\title{
A class number criterion for the equation $\left(x^{p}-1\right) /(x-1)=p y^{q}$
}

by

\section{Benjamin Dupuy (Bordeaux)}

1. Introduction. Let $p$ be an odd prime number and let

$$
\Phi(x)=\Phi_{p}(x)=\frac{x^{p}-1}{x-1}
$$

be the $p$ th cyclotomic polynomial. It is well-known that, for $x \in \mathbb{Z}$, the integer $\Phi(x)$ is divisible by at most the first power of $p$. More precisely, $p \nmid \Phi(x)$ if $x \not \equiv 1 \bmod p$, and $p \| \Phi(x)$ if $x \equiv 1 \bmod p$.

Indeed, if $p \mid \Phi(x)$ then $x^{p} \equiv 1 \bmod p$, which implies $x \equiv 1 \bmod p$. Now, using the binomial formula, we obtain

$\Phi(x)=\frac{(1+(x-1))^{p}-1}{x-1}=p+\sum_{k=2}^{p-1}\left(\begin{array}{l}p \\ k\end{array}\right)(x-1)^{k-1}+(x-1)^{p-1} \equiv p \bmod p^{2}$, which implies $p \| \Phi(x)$.

Let $q$ be another prime number. A classical Diophantine problem, studied, most recently, by Mihăilescu [6,7], is whether the $p$-free part of $\Phi(x)$ can be a $q$ th power. This can be rephrased as follows: given $e \in\{0,1\}$, does the equation $\Phi(x)=p^{e} y^{q}$ have a non-trivial solution in integers $x$ and $y$ ? (By trivial solutions we mean those with $x=e=0$ and $x=e=1$.)

The case $e=0$, that is, the equation $\Phi(x)=y^{q}$, is (a particular case of) the classical Nagell-Ljunggren equation. It is known to have several nontrivial solutions, and, as is commonly believed, no other solutions exist. See [3] for a comprehensive survey of results on this equation and methods for its analysis.

In the present note we study the case $e=1$, that is, the equation

$$
\frac{x^{p}-1}{x-1}=p y^{q} .
$$

(As we have seen above, any solution of this equation must satisfy $x \equiv 1$ $\bmod p$.)

2000 Mathematics Subject Classification: Primary 11D41; Secondary 11R18, 11S80. 
Let $h_{p}^{-}$be the $p$ th relative class number. Mihăilescu [7, Theorem 1] proved that (1) has no non-trivial solutions if $q \nmid h_{p}^{-}$and, in addition, some complicated technical condition involving $p$ and $q$ is satisfied. In this note we show that this technical condition is not required.

TheOREm 1.1. Let $p$ and $q$ be distinct odd prime numbers, $p \geq 5$. Assume that $q$ does not divide the relative class number $h_{p}^{-}$. Then (1) has no solutions in integers $x, y \neq 1$.

In particular, since $h_{p}^{-}=1$ for $p \leq 19$, equation (1) has no non-trivial solutions when $5 \leq p \leq 19$. (Neither does it have solutions when $p=3$, as was shown long ago by Nagell [8].)

The interest in equation (1) was inspired by the fact that it is closely related to the celebrated equation of Catalan $x^{p}-z^{q}=1$. In fact, Cassels [4] showed that any non-trivial solution of Catalan's equation gives rise to a solution of (1). All major contributions to the theory of Catalan's equation, including Mihăilescu's recent solution $[1,5]$, have Cassels' result as the starting point.

This article is strongly inspired by the work of Mihăilescu $[5,6,7]$. In particular, the argument in the case $q \not \equiv 1 \bmod p$ (see Section 6 ) can be found in [6]. However, the case $q \equiv 1 \bmod p$ (see Section 7) requires new ideas.

2. The cyclotomic field. Let $p$ be an odd prime number and let $\zeta=\zeta_{p}$ be a primitive $p$ th root of unity. In this section we collect several facts about the $p$ th cyclotomic field $K=\mathbb{Q}(\zeta)$. As usual, we denote by $K^{+}=K \cap \mathbb{R}=\mathbb{Q}(\zeta+\bar{\zeta})$ the maximal real subfield of $K$. (Here and below, $z \mapsto \bar{z}$ stands for the "complex conjugation" map.) We denote by $\mathcal{O}$ the ring of integers of $K$; it is well-known that $\mathcal{O}=\mathbb{Z}[\zeta]$.

We denote by $\mathfrak{p}$ the principal ideal $(1-\zeta)$. It is the only prime ideal of the field $K$ above $p$, and $\mathfrak{p}^{p-1}=(p)$. For $k \not \equiv l \bmod p$ the algebraic number

$$
\frac{\zeta^{k}-\zeta^{l}}{1-\zeta}
$$

is a unit of $K$ (called cyclotomic or circular unit); in other words, we have

$$
\left(\zeta^{k}-\zeta^{l}\right)=\mathfrak{p} .
$$

In particular,

$$
\zeta^{k}+\zeta^{l}=\frac{\zeta^{2 k}-\zeta^{2 l}}{1-\zeta} / \frac{\zeta^{k}-\zeta^{l}}{1-\zeta}
$$

is a unit in $K$. All this will be frequently used without special reference.

Finally, recall that $h_{p}^{+} \mid h_{p}$, where $h_{p}$ and $h_{p}^{+}$are the class numbers of $K$ and $K^{+}$, respectively, and the relative class number is defined by $h_{p}^{-}=h_{p} / h_{p}^{+}$. 
The proofs of all statements above can be found in the first chapters of any course of the theory of cyclotomic fields; see, for instance, [9].

The following observation provides a convenient tool for calculating traces of algebraic integers from $K$ modulo $p$. We denote by $\mathbb{F}_{p}$ the field of $p$ elements, and we let $\operatorname{Tr}: K \rightarrow \mathbb{Q}$ be the trace map.

Proposition 2.1. Let $\varrho: \mathcal{O} \rightarrow \mathbb{F}_{p}$ be the reduction modulo $\mathfrak{p}$. Then for any $a \in \mathcal{O}$ we have

$$
\varrho(a) \equiv-\operatorname{Tr}(a) \bmod p .
$$

Proof. We have $\varrho\left(\zeta^{n}\right)=1$ for all $n \in \mathbb{Z}$, and

$$
\operatorname{Tr}\left(\zeta^{n}\right)= \begin{cases}-1, & p \nmid n, \\ p-1, & p \mid n .\end{cases}
$$

Hence (2) holds for $a=\zeta^{n}$. By linearity, it extends to $\mathcal{O}=\mathbb{Z}[\zeta]$.

Here is an example of how one can use this.

Corollary 2.2. For any $u \in \mathbb{Z}$ put

$$
\chi_{u}=\frac{\zeta^{u}-\zeta}{\left(1+\zeta^{u}\right)(1-\zeta)} .
$$

Then

$$
2 \operatorname{Tr}\left(\chi_{u}\right) \equiv u-1 \bmod p
$$

In particular, $\operatorname{Tr}\left(\chi_{u}\right) \not \equiv 0 \bmod p$ unless $u \equiv 1 \bmod p$.

Proof. For $u \equiv 1 \bmod p$ we have $\chi_{u}=0$ and there is nothing to prove. Now let $u \not \equiv 1 \bmod p$. We may assume that $u>0$. We have

$$
\varrho\left(\frac{\zeta^{u}-\zeta}{1-\zeta}\right)=\varrho\left(-\zeta-\zeta^{2}-\cdots-\zeta^{u-1}\right)=1-u .
$$

Also, since $1+\zeta^{u}$ is a unit, we have

$$
\varrho\left(\frac{1}{1+\zeta^{u}}\right)=\varrho\left(1+\zeta^{u}\right)^{-1}=\frac{1}{2} .
$$

Hence $\varrho\left(\chi_{u}\right)=(1-u) / 2$, which implies $(5)$.

In the following example we cannot use (2) because the number we are interested in is not an algebraic integer.

Proposition 2.3. We have

$$
\operatorname{Tr}\left(\frac{\zeta}{(1-\zeta)^{2}}\right)=\frac{1-p^{2}}{12} .
$$

Proof. Consider the rational function

$$
F(t)=\sum_{k=1}^{p-1} \frac{\zeta^{k} t}{\left(1-\zeta^{k} t\right)^{2}} .
$$


Using (3), we obtain

$$
\begin{aligned}
F(t) & =-\sum_{k=1}^{p-1} \sum_{n=1}^{\infty} n \zeta^{k n} t^{n}=-\sum_{n=1}^{\infty} n \operatorname{Tr}\left(\zeta^{n}\right) t^{n} \\
& =\sum_{n=1}^{\infty} n t^{n}-p^{2} \sum_{n=1}^{\infty} n t^{p n}=-\frac{t}{(1-t)^{2}}+\frac{p^{2} t^{p}}{\left(1-t^{p}\right)^{2}} .
\end{aligned}
$$

When $t \rightarrow 1$ we have

$$
\begin{aligned}
\frac{t}{(1-t)^{2}} & =\frac{1}{(t-1)^{2}}+\frac{1}{t-1} \\
\frac{p^{2} t^{p}}{\left(1-t^{p}\right)^{2}} & =\frac{1}{(t-1)^{2}}+\frac{1}{t-1}+\frac{1-p^{2}}{12}+o(1) .
\end{aligned}
$$

Hence

$$
\operatorname{Tr}\left(\frac{\zeta}{(1-\zeta)^{2}}\right)=F(1)=\frac{1-p^{2}}{12}
$$

3. Binomial power series. We shall need a property of binomial power series in the non-archimedean domain. As usual, we denote by $\mathbb{Z}_{p}$ and $\mathbb{Q}_{p}$ the ring of $p$-adic integers and the field of $p$-adic numbers, and we extend the standard $p$-adic absolute value from $\mathbb{Q}_{p}$ to the algebraic closure $\overline{\mathbb{Q}}_{p}$.

Given $a \in \mathbb{Z}_{p}$, we let

$$
R_{a}(t)=(1+t)^{a}=1+a t+\left(\begin{array}{l}
a \\
2
\end{array}\right) t^{2}+\left(\begin{array}{l}
a \\
3
\end{array}\right) t^{3}+\cdots
$$

be the binomial power series. Its coefficients are $p$-adic integers, and for any $\tau$, algebraic over $\mathbb{Q}_{p}$ and with $|\tau|_{p}<1$, our series converges at $t=\tau$ in the field $\mathbb{Q}_{p}(\tau)$. For any $n=0,1, \ldots$ we have the obvious inequality

$$
\left|R_{a}(\tau)-\sum_{k=0}^{n}\left(\begin{array}{l}
a \\
k
\end{array}\right) \tau^{k}\right|_{p} \leq|\tau|_{p}^{n+1}
$$

When $a$ is $p$-adically small, a sharper inequality may hold. For instance,

$$
\left|R_{p}(\tau)-(1+p \tau)\right|_{p} \leq p|\tau|_{p}^{2}
$$

when $|\tau|_{p}$ is sufficiently small. We shall need a result of this kind for the second order Taylor expansion.

It will be convenient to use the familiar notation $O(\cdot)$ in a slightly nontraditional fashion: we say $\tau=O(v)$ if $|\tau|_{p} \leq|v|_{p}$.

Proposition 3.1. Assume $p \geq 5$ and that $|\tau| \leq p^{-1 /(p-3)}$. Then

$$
R_{a}(\tau)=1+a \tau-\frac{a}{2} \tau^{2}+O\left(a^{2} \tau^{2}\right)+O\left(a \tau^{3}\right)
$$


Proof. Since

$$
\frac{a(a-1)}{2} \tau^{2}=-\frac{a}{2} \tau^{2}+O\left(a^{2} \tau^{2}\right)
$$

equality (6) is an immediate consequence of

$$
R_{a}(\tau)=1+a \tau+\frac{a(a-1)}{2} \tau^{2}+O\left(a \tau^{3}\right)
$$

so it suffices to prove the latter.

We prove (7) by induction on the $p$-adic order of $a$. When $|a|_{p}=1$, equality (7) is an immediate consequence of the binomial formula (and holds even under the weaker assumption $|\tau|_{p}<1$ ). Now assume that (7) holds for some $a \in \mathbb{Z}_{p}$, and let us show that it holds with $a$ replaced by $p a$.

By the induction hypothesis, $R_{a}(\tau)=1+v$, where

$$
v=a \tau+\frac{a(a-1)}{2} \tau^{2}+O\left(a \tau^{3}\right) .
$$

Then

$$
\begin{aligned}
R_{p a}(\tau) & =(1+v)^{p}=1+p v+\frac{p(p-1)}{2} v^{2}+O\left(p v^{3}\right)+O\left(v^{p}\right) \\
& =1+p a \tau+\frac{p a(a-1)}{2} \tau^{2}+\frac{p a^{2}(p-1)}{2} \tau^{2}+O\left(p a \tau^{3}\right)+O\left((a \tau)^{p}\right) \\
& =1+p a \tau+\frac{p a(p a-1)}{2} \tau^{2}+O\left(p a \tau^{3}\right)+O\left((a \tau)^{p}\right)
\end{aligned}
$$

Since $|\tau| \leq p^{-1 /(p-3)}$, we have $\left|(a \tau)^{p}\right|_{p} \leq\left|p a^{p} \tau^{3}\right|_{p} \leq\left|p a \tau^{3}\right|_{p}$. Hence the term $O\left((a \tau)^{p}\right)$ in (8) can be disregarded. This completes the proof of $(7)$ and of the proposition.

4. A special unit of the cyclotomic field. We start the proof of Theorem 1.1. We fix, once and for all, distinct odd prime numbers $p$ and $q$, and rational integers $x, y \neq 1$ satisfying (1). Recall that

$$
x \equiv 1 \bmod p,
$$

this congruence being frequently used below without special reference. Also, we use without special reference the notation of Section 2.

In this section, we construct a special unit of the field $K$, which plays the central role in the proof of Theorem 1.1. Our starting point is the following well-known statement.

Proposition 4.1. Put

$$
\alpha=\frac{x-\zeta}{1-\zeta}
$$

Then we have the following: 
1. The principal ideal $(\alpha)$ is a qth power of an ideal of $K$.

2. Assume that $q$ does not divide the relative class number $h_{p}^{-}$. Then $\bar{\alpha} / \alpha$ is a qth power in $K$.

Though the proof can be found in the literature, we include it here for the reader's convenience. We closely follow [2].

Proof. Since

$\Phi_{p}(x)=(x-\zeta) \cdots\left(x-\zeta^{p-1}\right), \quad p=\Phi_{p}(1)=(1-\zeta) \cdots\left(1-\zeta^{p-1}\right)$, we may rewrite equation (1) as

$$
\prod_{k=1}^{p-1} \frac{x-\zeta^{k}}{1-\zeta^{k}}=y^{q} .
$$

Since $p=\mathfrak{p}^{p-1} \mid(x-1)$, we have $\mathfrak{p} \|\left(x-\zeta^{k}\right)$ for $k=1, \ldots, p-1$. Hence the numbers

$$
\alpha_{k}=\frac{x-\zeta^{k}}{1-\zeta^{k}} \quad(k=1, \ldots, p-1)
$$

are algebraic integers coprime with $\mathfrak{p}$.

On the other hand, since

$$
\left(1-\zeta^{k}\right) \alpha_{k}-\left(1-\zeta^{l}\right) \alpha_{l}=\zeta^{l}-\zeta^{k}
$$

the greatest common divisor of $\alpha_{k}$ and $\alpha_{l}$ should divide $\mathfrak{p}=\left(\zeta^{k}-\zeta^{l}\right)$. Hence the numbers $\alpha_{1}, \ldots, \alpha_{p-1}$ are pairwise coprime. (In particular, $\alpha$ and $\bar{\alpha}$ are coprime, to be used in the proof of Proposition 4.2.) Now (9) implies that each of the principal ideals $\left(\alpha_{k}\right)$ is a $q$ th power of an ideal. This proves part 1.

Now write $(\alpha)=\mathfrak{a}^{q}$, where $\mathfrak{a}$ is an ideal of $K$. If $q \nmid h_{p}^{-}$then the class of $\mathfrak{a}$ belongs to the real part of the class group. In other words, we have $\mathfrak{a}=\mathfrak{b}(\gamma)$, where $\gamma \in K^{*}$ and $\mathfrak{b}$ is a "real" ideal of $K$ (that is, $\mathfrak{b}=\overline{\mathfrak{b}}$ ). Further, $\mathfrak{b}^{q}$ is a principal real ideal; in other words, $\mathfrak{b}^{q}=(\beta)$, where $\beta \in K^{+}$. We obtain $(\alpha)=\left(\beta \gamma^{q}\right)$, that is, $\alpha$ is equal to $\beta \gamma^{q}$ times a unit of $K$.

Recall that if $\eta$ is a unit of a cyclotomic field then $\bar{\eta} / \eta$ is a root of unity. Since $\bar{\beta}=\beta$, we deduce that $\bar{\alpha} / \alpha$ is $(\bar{\gamma} / \gamma)^{q}$ times a root of unity. Since every root of unity in $K$ is a $q$ th power, we have shown that $\bar{\alpha} / \alpha$ is a $q$ th power. This proves part 2 .

From now on we assume that $q$ does not divide $h_{p}^{-}$. In particular, Proposition 4.1 implies that there exists $\mu \in K$ such that $\bar{\alpha} / \alpha=\mu^{q}$. Moreover, this $\mu$ is unique because $K$ does not contain non-trivial $q$ th roots of unity. Similarly, the field $K$ contains exactly one $q$ th root of $\alpha / \bar{\alpha}$. Since both $\bar{\mu}$ and $\mu^{-1}$ are $q$ th roots of $\alpha / \bar{\alpha}$, we have

$$
\mu^{-1}=\bar{\mu} \text {. }
$$

This will be used in Section 5 . 
Now we are ready to construct the promised unit.

Proposition 4.2. Let $u$ be the inverse of q modulo $p$ (that is, we have $u q \equiv 1 \bmod p)$. Then the algebraic number $\phi=\alpha\left(\mu+\zeta^{u}\right)^{q}$ is a unit of the field $K$.

Proof. Write the principal ideal $(\mu)$ as $\mathfrak{a} \mathfrak{b}^{-1}$, where $\mathfrak{a}$ and $\mathfrak{b}$ are co-prime integral ideals of $K$. Then $(\bar{\alpha} / \alpha)=\mathfrak{a}^{q} \mathfrak{b}^{-q}$. Moreover, since $\alpha$ and $\bar{\alpha}$ are coprime (see the proof of Proposition 4.1), we have $(\bar{\alpha})=\mathfrak{a}^{q}$ and $(\alpha)=\mathfrak{b}^{q}$.

Further, we have $\left(\mu+\zeta^{u}\right)=\mathfrak{c b}^{-1}$, where $\mathfrak{c}$ is yet another integral ideal of $K$. We obtain $(\phi)=\mathfrak{b}^{q} \mathfrak{c}^{q} \mathfrak{b}^{-q}=\mathfrak{c}^{q}$, which shows that $\phi$ is an algebraic integer.

Next, put

$$
\phi^{\prime}=\alpha^{q-1}\left(\sum_{k=0}^{q-1} \mu^{k}\left(-\zeta^{u}\right)^{q-1-k}\right)^{q} .
$$

The same argument as above proves that $\phi^{\prime}$ is an algebraic integer as well. Further,

$$
\phi \phi^{\prime}=\alpha^{q}\left(\left(\mu+\zeta^{u}\right) \sum_{k=0}^{q-1} \mu^{k}\left(-\zeta^{u}\right)^{q-1-k}\right)^{q}=\left(\alpha\left(\mu^{q}+\zeta^{u q}\right)\right)^{q} .
$$

Now recall that $\mu^{q}=\bar{\alpha} / \alpha$ and that $u q \equiv 1 \bmod p$. The latter congruence implies that $\zeta^{u q}=\zeta$, and we obtain

$$
\phi \phi^{\prime}=(\alpha(\bar{\alpha} / \alpha+\zeta))^{q}=(\bar{\alpha}+\zeta \alpha)^{q}=(1+\zeta)^{q} .
$$

Since $1+\zeta$ is a unit of $K$, so are $\phi$ and $\phi^{\prime}$.

5. An analytic expression for $\mu$. We shall work in the local field $K_{\mathfrak{p}}=\mathbb{Q}_{p}(\zeta)$. As before, we extend $p$-adic absolute value from $\mathbb{Q}_{p}$ to $K_{\mathfrak{p}}$, so that $|1-\zeta|_{p}=p^{-1 /(p-1)}$.

Since $p$ totally ramifies in $K$, every automorphism $\sigma$ of $K / \mathbb{Q}$ extends to an automorphism of $K_{\mathfrak{p}} / \mathbb{Q}_{p}$. In particular, the "complex conjugation" $z \mapsto \bar{z}$ extends to an automorphism of $K_{\mathfrak{p}} / \mathbb{Q}_{p}$ (we continue to call it "complex conjugation").

Let $R_{a}(t)$ be the binomial power series, introduced in Section 3. Since the automorphisms of $K_{\mathfrak{p}} / \mathbb{Q}_{p}$ (in particular the "complex conjugation") are continuous in the $\mathfrak{p}$-adic topology, for any $\tau \in K_{\mathfrak{p}}$ with $|\tau|_{p}<1$ and for any $\sigma \in \operatorname{Gal}\left(K_{\mathfrak{p}} / \mathbb{Q}_{p}\right)$ we have $R_{a}(\tau)^{\sigma}=R_{a}\left(\tau^{\sigma}\right)$. In particular, $\overline{R_{a}(\tau)}=R_{a}(\bar{\tau})$.

Put

$$
\lambda=\frac{x-1}{1-\zeta},
$$

so that

$$
\alpha=1+\lambda, \quad \bar{\alpha}=1+\bar{\lambda}=1-\zeta \lambda
$$


(recall that $\alpha$ is defined in Proposition 4.1). Then

$$
|\lambda|_{p}=|x-1|_{p} p^{1 /(p-1)} \leq p^{-(p-2) /(p-1)}<1,
$$

and similarly for $\bar{\lambda}$. In particular, for any $a \in \mathbb{Z}_{p}$, the series $R_{a}(t)$ converges at $t=\lambda$ and $t=\bar{\lambda}$.

We wish to express the quantity $\mu$, introduced in Section 4 , in terms of the binomial power series. Since both $\mu$ and $R_{1 / q}(\bar{\lambda}) R_{-1 / q}(\lambda)$ are $q$ th roots of $\bar{\alpha} / \alpha$, we have

$$
\mu=R_{1 / q}(\bar{\lambda}) R_{-1 / q}(\lambda) \xi,
$$

where $\xi \in K_{\mathfrak{p}}$ is a $q$ th root of unity. We want to show that $\xi=1$.

The field $\mathbb{Q}_{p}(\xi)$ is an unramified sub-extension of the totally ramified extension $K_{\mathfrak{p}}$. Hence $\mathbb{Q}_{p}(\xi)=\mathbb{Q}_{p}$, that is, $\xi \in \mathbb{Q}_{p}$. It follows that $\xi$ is stable with respect to all automorphisms of $K_{\mathfrak{p}} / \mathbb{Q}_{p}$; in particular, it is stable with respect to the "complex conjugation": $\bar{\xi}=\xi$.

Applying the "complex conjugation" to (11) and using (10), we obtain $\mu^{-1}=R_{1 / q}(\lambda) R_{-1 / q}(\bar{\lambda}) \xi$, which, together with (11), implies that $\xi^{2}=1$. Since $\xi$ is a $q$ th root of unity, this is possible only if $\xi=1$.

We have shown that

$$
\mu=R_{1 / q}(\bar{\lambda}) R_{-1 / q}(\lambda)=R_{1 / q}(-\zeta \lambda) R_{-1 / q}(\lambda) .
$$

The rest of the proof splits into two cases, depending on whether $q \not \equiv 1$ $\bmod p$ or $q \equiv 1 \bmod p$. The arguments in both cases are quite similar, but the latter case is technically more involved.

6. The case $q \not \equiv 1 \bmod p$. We have

$$
\mu=R_{1 / q}(-\zeta \lambda) R_{-1 / q}(\lambda)=1-\frac{1+\zeta}{q} \lambda+O\left(\lambda^{2}\right),
$$

where, as in Section 3, we say that $\tau=O(v)$ if $|\tau|_{p} \leq|v|_{p}$.

Hence, for the quantity $\phi$, introduced in Proposition 4.2, we have

$$
\begin{aligned}
\phi & =(1+\lambda)\left(1+\zeta^{u}-\frac{1+\zeta}{q} \lambda+O\left(\lambda^{2}\right)\right)^{q} \\
& =\left(1+\zeta^{u}\right)^{q}(1+\lambda)\left(1-\frac{1+\zeta}{1+\zeta^{u}} \lambda\right)+O\left(\lambda^{2}\right) \\
& =\left(1+\zeta^{u}\right)^{q}\left(1+\frac{\zeta^{u}-\zeta}{1+\zeta^{u}} \lambda\right)+O\left(\lambda^{2}\right) \\
& =\left(1+\zeta^{u}\right)^{q}\left(1+(x-1) \chi_{u}\right)+O\left(\lambda^{2}\right),
\end{aligned}
$$

where $\chi_{u}$ is defined in (4). 
Since the automorphisms of $K / \mathbb{Q}$ extend to automorphisms of $K_{\mathfrak{p}} / \mathbb{Q}_{p}$, the same is true for the norm and the trace maps: for any $a \in K$ we have

$$
\mathcal{N}_{K_{\mathfrak{p}} / \mathbb{Q}_{p}}(a)=\mathcal{N}_{K / \mathbb{Q}}(a), \quad \operatorname{Tr}_{K_{\mathfrak{p}} / \mathbb{Q}_{p}}(a)=\operatorname{Tr}_{K / \mathbb{Q}}(a) .
$$

Below, we shall simply write $\mathcal{N}(a)$ and $\operatorname{Tr}(a)$. Also, since the automorphisms are continuous, we have $|\mathcal{N}(a)|_{p} \leq|a|_{p}^{p-1}$ and $|\operatorname{Tr}(a)|_{p} \leq|a|_{p}$.

Taking the norm in (13), we obtain

$$
\mathcal{N}\left(\frac{\phi}{\left(1+\zeta^{u}\right)^{q}}\right)=1+(x-1) \operatorname{Tr}\left(\chi_{u}\right)+O\left(\lambda^{2}\right) .
$$

Since both $\phi$ and $1+\zeta^{u}$ are units, the norm on the left is \pm 1 . Since $-1 \not \equiv 1$ $\bmod p$, the norm is 1 , and we obtain $(x-1) \operatorname{Tr}\left(\chi_{u}\right)=O\left(\lambda^{2}\right)$.

But, since $q \not \equiv 1 \bmod p$, we also have $u \not \equiv 1 \bmod p$. Corollary 2.2 implies that $\operatorname{Tr}\left(\chi_{u}\right)$ is not divisible by $p$. We obtain

$$
|x-1|_{p} \leq|\lambda|_{p}^{2}=|x-1|_{p}^{2} p^{2 /(p-1)},
$$

which implies $|x-1|_{p} \geq p^{-2 /(p-1)}$. Since $p \mid(x-1)$, this is impossible as soon as $p \geq 5$.

This proves the theorem in the case $q \not \equiv 1 \bmod p$.

7. The case $q \equiv 1 \bmod p$. We have (12). Also, $u \equiv 1 \bmod p$ and $\chi_{u}=0$, which means that the first order Taylor expansions are no longer sufficient. We shall use the second order expansion. Put $a=(q-1) / q$, so that $|a|_{p} \leq p^{-1}$, and rewrite (12) as

$$
\mu=(1-\zeta \lambda) R_{-a}(-\zeta \lambda)(1+\lambda)^{-1} R_{a}(\lambda)
$$

For $p \geq 5$ we have

$$
|\lambda|_{p} \leq p^{-(p-2) /(p-1)} \leq p^{-1 /(p-3)},
$$

which means that Proposition 3.1 applies to $\tau=\lambda$. We obtain

$$
\begin{gathered}
R_{-a}(-\zeta \lambda)=1+a \zeta \lambda+\frac{\zeta^{2}}{2} a \lambda^{2}+O\left(a \lambda^{3}\right)+O\left(a^{2} \lambda^{2}\right), \\
R_{a}(\lambda)=1+a \lambda-\frac{a}{2} \lambda^{2}+O\left(a \lambda^{3}\right)+O\left(a^{2} \lambda^{2}\right) .
\end{gathered}
$$

Substituting this into (14), we get

$$
\begin{aligned}
\mu= & (1-\zeta \lambda)\left(1+a \zeta \lambda+\frac{a}{2} \zeta^{2} \lambda^{2}\right)(1+\lambda)^{-1}\left(1+a \lambda-\frac{a}{2} \lambda^{2}\right) \\
& +O\left(a \lambda^{3}\right)+O\left(a^{2} \lambda^{2}\right) \\
= & \left(1+(-\zeta+a+a \zeta) \lambda-\frac{(1+\zeta)^{2}}{2} a \lambda^{2}\right)(1+\lambda)^{-1} \\
& +O\left(a \lambda^{3}\right)+O\left(a^{2} \lambda^{2}\right) .
\end{aligned}
$$


It follows that

$$
\begin{aligned}
\phi= & (1+\lambda)(\mu+\zeta)^{q} \\
= & \left(1+(-\zeta+a+a \zeta) \lambda-\frac{(1+\zeta)^{2}}{2} a \lambda^{2}+\zeta(1+\lambda)\right)^{q}(1+\lambda)^{1-q} \\
& +O\left(a \lambda^{3}\right)+O\left(a^{2} \lambda^{2}\right) \\
= & (1+\zeta)^{q}\left(1+a \lambda-\frac{1+\zeta}{2} a \lambda^{2}\right)^{1+a /(1-a)}(1+\lambda)^{-a /(1-a)} \\
& +O\left(a \lambda^{3}\right)+O\left(a^{2} \lambda^{2}\right) .
\end{aligned}
$$

Applying Proposition 3.1 with the exponents $\pm a /(1-a)$ and taking into account the inequality $|a|_{p}<1$, we find

$$
\begin{aligned}
\left(1+a \lambda-\frac{1+\zeta}{2} a \lambda^{2}\right)^{a /(1-a)} & =1+\frac{a^{2}}{1-a} \lambda+O\left(a^{2} \lambda^{2}\right), \\
(1+\lambda)^{-a /(1-a)} & =1-\frac{a}{1-a} \lambda+\frac{a}{2(1-a)} \lambda^{2}+O\left(a \lambda^{3}\right) \\
& =1-\frac{a}{1-a} \lambda+\frac{a}{2} \lambda^{2}+O\left(a \lambda^{3}\right)+O\left(a^{2} \lambda^{2}\right) .
\end{aligned}
$$

Taking everything together, we obtain

$$
\begin{aligned}
\frac{\phi}{(1+\zeta)^{q}}= & \left(1+a \lambda-\frac{1+\zeta}{2} a \lambda^{2}\right)\left(1+\frac{a^{2}}{1-a} \lambda\right)\left(1-\frac{a}{1-a} \lambda+\frac{a}{2} \lambda^{2}\right) \\
& +O\left(a \lambda^{3}\right)+O\left(a^{2} \lambda^{2}\right) \\
= & 1-\frac{\zeta}{2} a \lambda^{2}+O\left(a \lambda^{3}\right)+O\left(a^{2} \lambda^{2}\right) \\
= & 1-\frac{\zeta}{2(1-\zeta)^{2}} a(x-1)^{2}+O\left(a \lambda^{3}\right)+O\left(a^{2} \lambda^{2}\right) .
\end{aligned}
$$

Now we complete the proof in the same fashion as in Section 6. Taking the norm, we find

$$
\pm 1=1-\frac{1}{2} \operatorname{Tr}\left(\frac{\zeta}{(1-\zeta)^{2}}\right) a(x-1)^{2}+O\left(a \lambda^{3}\right)+O\left(a^{2} \lambda^{2}\right) .
$$

The -1 on the left is again impossible, and if we have 1 , then, in view of Proposition 2.3, we must have the inequality

$$
\begin{aligned}
|x-1|_{p}^{2} & \leq \max \left\{|\lambda|_{p}^{3},|a|_{p}|\lambda|_{p}^{2}\right\} \\
& =\max \left\{|x-1|_{p}^{3} p^{3 /(p-1)},|a|_{p}|x-1|_{p}^{2} p^{2 /(p-1)}\right\},
\end{aligned}
$$

which means that either $|x-1|_{p} \geq p^{-3 /(p-1)}$ or $|a|_{p} \geq p^{-2 /(p-1)}$. But, for $p \geq 5$, neither of the latter inequalities can hold, because $|x-1|_{p} \leq p^{-1}$ and $|a|_{p} \leq p^{-1}$. The theorem is proved in the case $q \equiv 1 \bmod p$ as well. 
Acknowledgments. I thank Yuri Bilu for helping me to polish the exposition, and for indicating and correcting a mistake in the previous version of the proof. I also thank Professor P. Mihăilescu for a useful discussion, and Professor A. Schinzel for a helpful suggestion.

\section{References}

[1] Y. Bilu, Catalan's conjecture (after Mihăilescu), Séminaire Bourbaki, Vol. 2002-2003, Exp. 909, Astérisque 294 (2004), 1-26.

[2] Y. Bilu, Y. Bugeaud and M. Mignotte, The Problem of Catalan, Springer, to appear.

[3] Y. Bugeaud et M. Mignotte, L'équation de Nagell-Ljunggren $\left(x^{n}-1\right) /(x-1)=y^{q}$, Enseign. Math. (2) 48 (2002), 147-168.

[4] J. W. S. Cassels, On the equation $a^{x}-b^{y}=1$, II, Proc. Cambridge Philos. Soc. 56 (1960), 97-103; corrigendum, ibid. 57 (1961), 187.

[5] P. Mihăilescu, Primary cyclotomic units and a proof of Catalan's conjecture, J. Reine Angew. Math. 572 (2004), 167-195.

[6] - On the class groups of cyclotomic extensions in presence of a solution to Catalan's equation, J. Number Theory 118 (2006), 123-144.

[7] - New bounds and conditions for the equation of Nagell-Ljunggren, J. Number Theory, to appear.

[8] T. Nagell, Des équations indéterminées $x^{2}+x+1=y^{n}$ et $x^{2}+x+1=3 y^{n}$, Norsk Matem. Forenings Skrifter I, 2 (1921), 14 pp.; see also: Collected Papers of Trygve Nagell, ed. by P. Ribenboim, Vol. 1, Queen's Papers in Pure and Appl. Math. 121, Kingston, 2002, 79-94.

[9] L. Washington, Introduction to Cyclotomic Fields, 2nd ed., Grad. Texts in Math. 83, Springer, New York, 1997.

Institut de Mathématiques

Université Bordeaux 1

351 cours de la Libération

33405 Talence, France

E-mail: Benjamin.Dupuy@math.u-bordeaux1.fr

Received on 21.9.2006

and in revised form on 4.1.2007 